\title{
On the Number of Cusps of Rational Cuspidal Plane Curves
}

Jens Piontkowski

\section{CONTENTS}

\section{Introduction}

2. How to Obtain Theorem 1.3

References

2000 AMS Subject Classification: Primary 14H20, 14H45, 14H50

Keywords: Plane curves, rational curves, singularities, cusps
A cuspidal curve is a curve whose singularities are all cusps, i.e., unibranched singularities. This article describes computations that lead to the following conjecture: A rational cuspidal plane curve of degree greater than or equal to six has at most three cusps. The curves with precisely three cusps occur in three series. Assuming the Flenner-Zaidenberg rigidity conjecture, the above conjecture is verified up to degree 20 .

\section{INTRODUCTION}

Let $C \subset \mathbb{P}^{2}$ be a plane curve. The curve is called cuspidal if all its singularities are cusps, i.e., unibranched singularities. It is known that rational cuspidal plane curves exhibit severe restrictions. For example, Tono proved [Tono 05] the following theorem:

Theorem 1.1. A rational cuspidal plane curve has at most eight cusps.

It is not known whether this bound is sharp. In fact, one of the main purposes of this article is to provide evidence for the following conjecture:

Conjecture 1.2. A rational cuspidal plane curve has at most three cusps, with the exception of a rational plane quintic with four cusps.

We will describe the topological type of a cusp singularity by its multiplicity sequence $\bar{m}=\left(m_{0}, m_{1}, \ldots, m_{n}\right)$ : Let

$$
\begin{aligned}
& V:=X_{n} \quad \stackrel{\pi_{n}}{\rightarrow} X_{n-1} \quad \rightarrow \quad \cdots \quad \rightarrow \quad X_{1} \stackrel{\pi_{1}}{\rightarrow} \quad X_{0}=\left(\mathbb{C}^{2}, 0\right) \\
& \stackrel{\cup}{C_{n}} \rightarrow \underset{C_{n-1}}{\cup} \rightarrow \cdots \rightarrow \stackrel{\cup}{\cup} \rightarrow \stackrel{\cup}{C_{0}}=(C, p)
\end{aligned}
$$

be the minimal embedded resolution of the curve germ $(C, p)$ such that the total inverse image $D:=\pi_{n}^{-1} \circ \cdots \circ$ $\pi_{1}^{-1}(C)$ of the curve is a normal crossing divisor. Then the multiplicity sequence consists of the multiplicities $m_{i}$

(c) A K Peters, Ltd. 1058-6458/2007 \$0.50 per page Experimental Mathematics 16:2, page 251 
of the strict transforms $C_{i}$ of the curve germs. For example, a simple cusp has the multiplicity sequence $(2,1,1)$. To shorten the notation, sequences of the same multiplicity in the multiplicity sequence are indicated by subscripts, e.g., $\left(7,2_{3}, 1_{2}\right)$ means $(7,2,2,2,1,1)$. (Here we follow the notation of [deJong and Pfister 00] and [Matsuoka and Sakai 89], i.e., the number of 1's at the end of the multiplicity sequence equals the smallest number greater than one in the multiplicity sequence. Note that [Flenner and Zaidenberg 96, Flenner and Zaidenberg 00] add an additional 1.) The ending sequence of 1's is usually dropped from the notation, because it can be recovered from the remainder.

In this article we are hunting for cuspidal curves with the maximal number of cusps. The known rational cuspidal plane curves with at least three cusps are these:

1. ([Matsuoka and Sakai 89, Section 4] or [Namba 84]) For rational cuspidal plane curves of degree 5, the following combinations of cusps are possible: $\left[(3),\left(2_{2}\right),(2)\right],\left[\left(2_{2}\right),\left(2_{2}\right),\left(2_{2}\right)\right]$, and $\left[\left(2_{3}\right),(2),(2),(2)\right]$.

2. [Flenner and Zaidenberg 96, Theorem 3.5] For any $d \geq 4, n \geq m \geq 1$ with $n+m=d-2$, there is a rational cuspidal plane curve $C$ of degree $d$ with the three cusps $\left[(d-2),\left(2_{n}\right),\left(2_{m}\right)\right]$.

3. [Flenner and Zaidenberg 00, Theorem 1.1] For any $d=2 k+3, k \geq 1$, there is a rational cuspidal plane curve $C$ of degree $d$ with the three cusps $\left[\left(d-3,2_{k}\right),\left(3_{k}\right),(2)\right]$.

4. [Fenske 99, Section 4] For any $d=3 k+4, k \geq 1$, there is a rational cuspidal plane curve $C$ of degree $d$ with the three cusps $\left[\left(d-4,3_{k}\right),\left(4_{k}, 2_{2}\right),(2)\right]$.

All the above curves are unique up to projective equivalence. Not only is the existence of these curves known, but explicit constructions of them as well. Flenner, Zaidenberg, and Fenske described how to successively obtain the above curves in items 2,3 , and 4 by plane Cremona transformations starting from three curves given by equations.

Flenner and Zaidenberg proved more: Assuming that a rational cuspidal plane curve $C$ of degree $d \geq 6$ has at least three cusps and a point of multiplicity $d-2$ or $d-3$, then it is one of the curves in item 2 or 3 above. Fenske showed the analogous statement with $d-4$ for the curves in item 4 under the additional assumption of $\gamma_{2}=0$. This condition is explained below and is, for example, implied by the (unproven) Flenner-Zaidenberg rigidity conjecture.

The purpose of this article is to explain how to obtain by computer computations the following theorem:

Theorem 1.3. If $C \subseteq \mathbb{P}^{2}$ is a rational cuspidal plane curve of degree $d \leq 20$ with at least three cusps and $\gamma_{2}=0$, then it is contained in the above list.

One is immediately led to conjecture the following:

Conjecture 1.4. Any rational cuspidal plane curve with at least three cusps is contained in the above list.

Finally, we explain the condition $\gamma_{2}=0$. Let $(V, D)$ be the minimal embedded resolution of $C \subset \mathbb{P}^{2}$, which was described above locally. Here $D$ is a normal crossing divisor endowed with its reduced structure. The deformations of the pair $(V, D)$ correspond to equisingular deformations of the curve $C \subset \mathbb{P}^{2}$ [Flenner and Zaidenberg 96, Sections 1.6-2.1]. Let $\Theta_{V}\langle D\rangle$ denote the logarithmic tangent bundle. The infinitesimal automorphism group of the pair $(V, D)$ is $H^{0}\left(V, \Theta_{V}\langle D\rangle\right)$, the infinitesimal deformations are $H^{1}\left(V, \Theta_{V}\langle D\rangle\right)$, and the obstruction space for these deformations is $H^{2}\left(V, \Theta_{V}\langle D\rangle\right)$.

We will assume throughout the article that the curve $C$ has at least three cusps. Then $X:=\mathbb{P}^{2} \backslash C$ is of log-general type [Wakabayashi 78]. By Iitaka's theorem [Iitaka 77, Theorem 6], the automorphism group of a log-general surface is finite. Thus $H^{0}\left(V, \Theta_{V}\langle D\rangle\right)=0$. The pair $(V, D)$ or the curve $C$ is called rigid, respectively unobstructed, if $h^{1}\left(V, \Theta_{V}\langle D\rangle\right)=0$, respectively $h^{2}\left(V, \Theta_{V}\langle D\rangle\right)=0$. Flenner and Zaidenberg computed the Euler characteristic of the logarithmic tangent bundle as

$$
\begin{aligned}
\chi\left(\Theta_{V}\langle D\rangle\right) & =-h^{1}\left(V, \Theta_{V}\langle D\rangle\right)+h^{2}\left(V, \Theta_{V}\langle D\rangle\right) \\
& =K_{V}\left(K_{V}+D\right)
\end{aligned}
$$

where $K_{V}$ is the canonical divisor of $V$. They conjectured in an even more general setting that $(D, V)$ is always rigid and unobstructed. In our special situation, Tono [Tono 05, Lemma 4.1 and Remark 4.5] showed that

$$
\gamma_{2}:=h^{0}\left(2\left(K_{V}+D\right)\right)=K_{V}\left(K_{V}+D\right),
$$

and in particular that $\gamma_{2}=\chi\left(\Theta_{V}\langle D\rangle\right) \geq 0$ and unobstructedness implies rigidity. 


\section{HOW TO OBTAIN THEOREM 1.3}

The basic idea is to produce enough combinatorial restrictions on the multiplicity sequences of the cusps on a rational plane curve such that only the curves in the list remain as possible curves. These restrictions will be checked by a computer program [Piontkowski 07].

The first step of the program is to produce all possible multiplicity sequences of cusps that might occur on a curve of degree $d$. By [Flenner and Zaidenberg 96, Proposition 1.2], a sequence of positive integers $\bar{m}=$ $\left(m_{0}, m_{1}, \ldots, m_{n}\right)$ is the multiplicity sequence of a cusp $p$ if and only if for each $i=1, \ldots, n$ there is a $k \geq 0$ such that

$$
m_{i-1}=m_{i}+\cdots+m_{i+k}
$$

where

$$
m_{i}=m_{i+1}=\cdots=m_{i+k-1},
$$

and the number of ending 1's equals the smallest $m_{i}$ greater than 1.

The resulting curve has to be rational; hence it has to pass the following test:

$\star \delta$ Test: The $\delta$ invariant of a cusp $p$ is $\delta_{p}=$ $\sum_{i=0}^{n} m_{i}\left(m_{i}-1\right) / 2$. On a rational curve, the sum of the $\delta$ invariants of the cusps equals the arithmetic genus of $C$, which is $(d-1)(d-2) / 2$. (See, for example, [Orevkov and Zaidenberg 95, Lemma 4].)

In particular, for any cusp we have $m_{0} \leq d-1$. In fact, $m_{0} \leq d-2$ because we want to have more than one singularity on $C$.

By Bézout's theorem, the tangent line $\mathbb{T}_{p} C$ intersects $C$ in $p$ with multiplicity at most $d$, i.e., $m_{0}+m_{1} \leq d$. However, if $m_{0}+m_{1}=d$, then $\mathbb{T}_{p} C$ intersects $C$ only in $p$, and by the Lin-Zaidenberg theorem [Lin and Zaidenberg 78], the affine curve $C \backslash\{p\} \subset \mathbb{P}^{2} \backslash \mathbb{T}_{p} C=\mathbb{C}^{2}$ is biregular to a curve of the form $x^{l}-y^{k}=0$ for some $k, l \in \mathbb{N}$ with $\operatorname{gcd}(l, k)=1$. In this case, $C$ has at most two cusps, and thus in our case we must have $m_{0}+m_{1}<d$.

After producing the list of singularities that might occur on a rational cuspidal curve with at least three cusps, the program starts to search for possible combinations of singularities. First, it searches by a backtracking algorithm for combinations of three to eight cusps such that the sum of their $\delta$ invariants equals the arithmetic genus, thereby implying that the resulting curve is rational. To these combinations the following tests are applied, which were used by Flenner and Zaidenberg: $\star \gamma_{2}$ test. We examine only curves with $\gamma_{2}=0$, where $\gamma_{2}$ can be computed as

$$
\gamma_{2}=3(3-d)+\sum_{p \in \operatorname{Sing} C}\left(\eta_{p}+\omega_{p}-2\right),
$$

where $\eta_{p}$ is defined for a cusp $p$ with multiplicity sequence $\bar{m}$ by

$$
\eta_{p}=\sum_{i=0}^{n}\left(m_{i}-1\right)
$$

and $\omega_{p}$ is the number of subdivisional blowups in the minimal resolution of the cusp $p$ [Flenner and Zaidenberg 96, Corollary 2.5]. A blowup is called subdivisional if it is performed at an intersection point of exceptional divisors of previous blowups; otherwise, it is called sprouting. The exception is the first blowup, which is subdivisional by definition. (Note that we follow the definition of [Matsuoka and Sakai 89], where the $\omega_{p}$ is one bigger than that of [Flenner and Zaidenberg 96, Flenner and Zaidenberg 00].) In terms of the multiplicity sequence, the number $\omega_{p}$ can be computed as ([Flenner and Zaidenberg 96, Lemma 2.3] or [Matsuoka and Sakai 89, Lemma 1])

$$
\omega_{p}=\sum_{i=1}^{n}\left(\left\lceil\frac{m_{i-1}}{m_{i}}\right\rceil-1\right)+1
$$

* Bézout test. Each line in the plane intersects the curve in at most $d$ points, counting multiplicities. In particular, for a line through the cusps $p, q$, the first elements of their multiplicity sequences $\bar{m}^{p}$ and $\bar{m}^{q}$ must satisfy

$$
m_{0}^{p}+m_{0}^{q} \leq d
$$

$\star$ Hurwitz test. Let $p_{1}, \ldots, p_{r}$ denote the cusps of the curve and $\bar{m}^{p_{i}}$ their multiplicity sequences. The projection from $p_{i}$ is a $d-m_{0}^{p_{i}}$ covering $C \cong \mathbb{P}^{1} \rightarrow \mathbb{P}^{1}$, to which we apply the Riemann-Hurwitz formula. This yields

$$
2\left(d-m_{0}^{p_{i}}\right)-2=\sum_{q \in C}\left(\nu_{q}-1\right),
$$

where $\nu_{q}$ is the ramification index of the covering in $q \in C$. For the test we use the estimates $\nu_{p_{i}} \geq m_{1}^{p_{i}}$ and $\nu_{p_{j}} \geq m_{0}^{p_{j}}$ for $j \neq i$. Thus

$$
\sum_{j=1}^{r}\left(m_{0}^{p_{j}}-1\right)+m_{0}^{p_{i}}+m_{1}^{p_{i}} \leq 2 d-2 .
$$


These tests sum up the method of Flenner and Zaidenberg for the classification of rational cuspidal plane curves with at least three cusps and among them a cusp of multiplicity $d-2$ or $d-3$. They are also enough to prove our theorem for $d \in\{6,8,10,14,16\}$ : The program described above produced only the known combinations of singularities in these cases. For the other degrees, some additional combinations appeared. The easiest way to reduce the number of possible combinations further is to apply two propositions of Fenske. He observed that if the curve has a cusp whose multiplicity sequence starts like $[\lfloor d / 2\rfloor,\lfloor d / 2\rfloor,\lfloor d / 2\rfloor, \ldots]$ for odd $d$ or $[d / 2, d / 2, d / 2, \ldots]$ for even $d$, then it can be transformed into another rational cuspidal plane curve by a plane Cremona transformation. More precisely, his results are as follows:

1. [Fenske 99, Lemma 4.1.13 and Corollary 4.1.14] A rational cuspidal curve $C$ of degree $d=2 k+1$ and cusp type $\left[\left(k, k, k, \bar{m}_{1}\right), \bar{m}_{2}, \ldots, \bar{m}_{r}\right]$ can be taken by a Cremona transformation to a rational cuspidal curve $C^{\prime}$ of degree $d^{\prime}=k+2$ and cusp type $\left[\bar{m}_{1}, \bar{m}_{2}, \ldots, \bar{m}_{r}\right]$. Further, the curve $C^{\prime}$ has a regular point $q$ whose tangent line cuts $C^{\prime}$ with multiplicity 2 in $q$ and with multiplicity $k$ in the cusp of type $\bar{m}_{1}$.

Rational cuspidal curves of degree $d=2 k+1$ and cusp type $\left[\left(k, k, k, k, \bar{m}_{1}\right), \bar{m}_{2}, \ldots, \bar{m}_{r}\right]$ with $r \geq 3$ and $\bar{m}_{2}, \ldots, \bar{m}_{r}$ nontrivial do not exist.

2. [Fenske 99, Lemma 4.1.15] A rational cuspidal curve $C$ of degree $d=2 k$ and cusp type $\left[\left(k, k, k, \bar{m}_{1}\right), \bar{m}_{2}, \ldots, \bar{m}_{r}\right]$ can be taken by a Cremona transformation to a rational cuspidal curve $C^{\prime}$ of degree $d^{\prime}=k$ and cusp type $\left[\bar{m}_{1}, \bar{m}_{2}, \ldots, \bar{m}_{r}\right]$. Further, the tangent line to $C^{\prime}$ in the cusp of type $\bar{m}_{1}$ intersects $C^{\prime}$ only in this cusp (with multiplicity $k$ ).

This excludes the following numbers of cases:

\begin{tabular}{c|cccccccc}
$d$ & 7 & 9 & 11 & 12 & 13 & 15 & 17 & 19 \\
\hline number of cases & 3 & 5 & 9 & 2 & 16 & 24 & 6 & 52
\end{tabular}

This deals with most previously unknown combinations. The remaining ones are listed in Table 1, together with the name of a test that shows that it cannot exist. We will describe these tests below.

Here $X_{3}$ and $X_{4}$ stand for one of the following combinations of cusps singularities:

$$
\begin{aligned}
X_{3} \in\left\{\left[\left(2_{2}\right),(2)\right],[(2),(2),(2)]\right\}, \\
X_{4} \in\left\{[(3),(2)],\left[\left(2_{3}\right),(2)\right],\left[\left(2_{2}\right),\left(2_{2}\right)\right],\left[\left(2_{2}\right),(2),(2)\right],\right. \\
\\
{[(2),(2),(2),(2)]\} . }
\end{aligned}
$$

\begin{tabular}{c|l|l}
$d$ & Cusp Type & Test \\
\hline 13 & {$\left[\left(8,4_{6}\right),(2),(2)\right]$} & $\Gamma$ test with $l=3$ for $\left(8,4_{6}\right)$ \\
\hline 17 & {$\left[\left(7_{5}, 5,2_{2}\right), X_{3}\right]$} & $\Gamma$ test with $l=2$ for $\left(7_{5}, 5,2_{2}\right)$ \\
\hline 18 & {$\left[\left(8_{4}, 6,2_{7}\right),(2),(2)\right]$} & $\Gamma$ test, $l=2$ for $\left(8_{4}, 6,2_{7}\right)$ \\
18 & {$\left[\left(8_{4}, 6,2_{6}\right), X_{3}\right]$} & $\Gamma$ test, $l=2$ for $\left(8_{4}, 6,2_{6}\right)$ \\
18 & {$\left[\left(8_{4}, 6,2_{5}\right), X_{4}\right]$} & $\Gamma$ test $l=2$ for $\left(8_{4}, 6,2_{5}\right)$ \\
18 & {$\left[\left(8_{4}, 6,2_{4}\right), \ldots\right]$} & $\Gamma$ test, $l=2$ for $\left(8_{4}, 6,2_{4}\right)$ \\
18 & {$\left[\left(8_{4}, 6_{3}\right), \ldots\right]$} & $\Gamma$ test, $l=2$ for $\left(8_{4}, 6,2_{3}\right)$ \\
18 & {$\left[\left(8_{4}, 4_{3}, 3\right), X_{3}\right]$} & double covering $/$ spectrum test \\
18 & {$\left[\left(8_{4}, 4_{3}, 2_{4}\right),(2),(2)\right]$} & double covering $/$ spectrum test \\
18 & {$\left[\left(8_{4}, 4_{3}, 2_{3}\right), X_{3}\right]$} & double covering $/$ spectrum test \\
18 & {$\left[\left(8_{4}, 4_{3}, 2_{2}\right), X_{4}\right]$} & double covering $/$ spectrum test \\
\hline 19 & {$\left[\left(12,6_{5}, 3_{3}, 2\right),(2),(2)\right]$} & spectrum test \\
19 & {$\left[\left(12,6_{5}, 3_{3}\right), X_{3}\right]$} & spectrum test \\
19 & {$\left[\left(12,6_{5}, 4,2_{4}\right),(2),(2)\right]$} & $\Gamma$ test, $l=3$ for $\left(12,6_{5}, 4,2_{4}\right)$ \\
19 & {$\left[\left(12,6_{5}, 4,2_{3}\right), X_{3}\right]$} & $\Gamma$ test, $l=3$ for $\left(12,6_{5}, 4,2_{3}\right)$ \\
19 & {$\left[\left(12,6_{5}, 4,2_{2}\right), X_{4}\right]$} & $\Gamma$ test, $l=3$ for $\left(12,65,4,2_{2}\right)$ \\
\hline 20 & {$\left[\left(9_{4}, 5,4\right),(5),(2)\right]$} & $\Gamma$ test with $l=2$ for $\left(9_{4}, 5,4\right)$ \\
20 & {$\left[\left(8_{6}\right), X_{3}\right]$} & spectrum test
\end{tabular}

TABLE 1. The difficult cases.

The proof of the following simple test is based on Bézout's theorem:

Proposition 2.1. ( $\Gamma$ test.) [de Bobadilla et al. 06, Section 3.4, Proposition 2] Let $\Gamma_{i}$ be the semigroup rings of the $r$ cusps $p_{i}$ on a cuspidal plane curve of degree $d$. Then for all $l<d$ and $n_{i} \in \mathbb{N}_{0}$ with $\sum n_{i} \geq l d$ the following inequalities hold:

$$
\sum_{i=1}^{r} \#\left(\Gamma_{i} \cap\left[0, n_{i}\right]\right) \geq \frac{(l+1)(l+2)}{2} .
$$

For the combinations in the table where this method is indicated, it was enough to apply this proposition to the worst singularity alone to see that it cannot occur on a curve of that particular degree.

Another known method to exclude some combinations of singularities is through the cyclic covering method ([Matsuoka and Sakai 89, Section 3] or [Yoshihara 97, Yoshihara 89]). It works as follows: Assume that a plane curve $C$ with a certain combination of singularities exists. One constructs a surface $S$ as the minimal resolution of a cyclic covering of $\mathbb{P}^{2}$ branched along the curve $C$ and then shows that this surface has impossible invariants. Even using the algorithm of Némethi to construct the resolution of the covering [Némethi 96, Section 5], this is a very laborious method; it cannot deal with all the remaining cases.

The most powerful method seems to be provided by the semicontinuity of the spectrum ([de Bobadilla et al. 06, Section 3.6] or [Kulikov 98, Chapter II, Section 8.9]). If $\left(C, p_{i}\right)$ are the singularities of a plane curve $C$ of degree $d$, then the multisingularity $\sum_{i}\left(C, p_{i}\right)$ is a defor- 
mation of the plane germ $\left(x^{d}+y^{d}, 0\right)$. Therefore, the union of the spectra of the singularities $\left(C, p_{i}\right)$ satisfies the semicontinuity property compared with the spectrum of $\left(x^{d}+y^{d}, 0\right)$ for any interval $(\alpha, \alpha+1]$. The spectrum of a plane cusp can be computed by purely combinatorial means using Saito's formula [Saito 00]. The author implemented this formula into Singular [Greuel et al. 05] as part of the library cusp.lib, which extends the built-in library gmssing. lib [Piontkowski 07]. With the help of this library such checks can easily be done.

\section{REFERENCES}

[de Bobadilla et al. 06] J. Fernández de Bobadilla, I. LuengoVelasco, A. Melle-Hernández, and A. Némethi. "On Rational Cuspidal Projective Plane Curves." Proc. London Math. Soc. (3) 92:1 (2006), 99-138.

[deJong and Pfister 00] T. deJong and G. Pfister. Local Analytic Geometry. Braunschweig: Vieweg, 2000.

[Fenske 99] T. Fenske. "Unendliche Serien ebener rationaler kuspidaler Kurven vom Typ $(d, d-k)$." PhD diss., University of Bochum, 1999.

[Flenner and Zaidenberg 96] H. Flenner and M. Zaidenberg. "On a Class of Rational Cuspidal Plane Curves." Manuscripta Math. 89 (1996), 439-459.

[Flenner and Zaidenberg 00] H. Flenner and M. Zaidenberg. "Rational Cuspidal Plane Curves of Type $(d, d-3) . "$ Math. Nachr. 210 (2000), 93-110.

[Greuel et al. 05] G.-M. Greuel, G. Pfister, and H. Schönemann. "Singular 3.0." Software available online from the University of Kaiserslautern (http://www.singular.uni-kl. de), 2005.

[Iitaka 77] S. Iitaka. "On Logarithmic Kodaira Dimension of Algebraic Varieties." In Complex Analysis and Algebraic Geometry, pp. 175-189. Tokyo: Iwanami Shoten, 1977.
[Kulikov 98] V. Kulikov. Mixed Hodge Structures and Singularities, Cambridge Tracts in Math. 132. Cambridge: Cambridge Univ. Press, 1998.

[Lin and Zaidenberg 78] V. Lin and M. Zaidenberg. "An Irreducible Simply Connected Curve in $\mathbb{C}^{2}$ Is Equivalent to a Quasihomogeneous Curve." Soviet Math. Dokl. 28 (1978), 200-204.

[Matsuoka and Sakai 89] T. Matsuoka and F. Sakai. "The Degree of Rational Cuspidal Curves." Math. Ann. 285 (1989), 233-247.

[Namba 84] M. Namba. Geometry of Projective Algebraic Curves. New York: Dekker 1984.

[Némethi 96] A. Némethi. "The Signature of $f(x, y)+z^{N}$." In Singularity Theory (Liverpool, 1996), pp. 131-149, London Math. Soc. Lecture Note Ser. 263. Cambridge: Cambridge Univ. Press, 1999.

[Orevkov and Zaidenberg 95] S. Orevkov and M. Zaidenberg. "On the Number of Singular Points of Plane Curves." arXiv:alg-geom/9507005, 1995.

[Piontkowski 07] Jens Piontkowski. Computer program available online (http://www.expmath.org/expmath/volumes/ 16/16.2/Piontkowski/supplement.tar), 2007.

[Saito 00] M. Saito. "Exponents of an Irreducible Plane Curve Singularity." arXiv: math.AG/0009133, 2000.

[Tono 05] K. Tono. "On the Number of the Cusps of Cuspidal Plane Curves." Math. Nachr. 278 (2005), 216-221.

[Wakabayashi 78] I. Wakabayashi. "On the Logarithmic Kodaira Dimension of the Complement of a Curve in $\mathbb{P}^{2}$." Proc. Japan Acad. 54A (1978), 157-162.

[Yoshihara 89] H. Yoshihara. "Plane Curves Whose Singular Points Are Cusps and Triple Coverings of $\mathbb{P}^{2}$." Manuscripta Math. 64 (1989), 169-187.

[Yoshihara 97] H. Yoshihara. "A Note on the Existence of Some Curves." In Algebraic Geometry and Commutative Algebra in Honor of M. Nagata, edited by H. Hijikata et al., pp. 801-804. Tokyo: Kinokuniya, 1997.

Jens Piontkowski, Mathematisches Institut, Heinrich-Heine-Universität Düsseldorf, Universitätsstr. 1, 40225 Düsseldorf, Germany (piontkow@uni-duesseldorf.de) 